\title{
Practice and Research of HTC Vive Controller Functions in Virtual Reality Interaction
}

\author{
Lei Chen \\ Department of Computer Science and Engineering \\ Southern University of Science and Technology \\ Shenzhen, China \\ chen13@mail.sustc.edu.cn
}

\author{
Zongwei Luo \\ Department of Computer Science and Engineering \\ Southern University of Science and Technology \\ Shenzhen, China \\ luozw@sustc.edu.cn
}

\begin{abstract}
Virtual Reality has been a state-of-the-art technology recently, and HTC Vive is one of the best sellers that is based on this technology with its own controllers which are one of its features. This article starts with a series of development projects of virtual reality, develops some influential functions of Vive controllers, and carries out researches from the practical effect. Results show that powerful functions of the controllers is the core part in the VR interaction effect, and the distance between virtual world and real world can be constantly shrunk benefiting from the breach.
\end{abstract}

Keywords-Virtual Reality interaction; HTC Vive; controller function; project practice and research.

\section{BACKGROUND INTRODUCTION}

The burst of virtual reality technology has attracted many large companies in this world, and VR products have emerged with the trend: The Facebook Oculus Rift, Samsung Gear VR device, HTC Vive from HTC and Valve, and SONY's Morpheus VR head mount display[1]. Among the list, the HTC Vive gives the players more interaction with the contents, so there is a low level of otherness between visual and vestibular systems which leads to a relatively better experience and less motion sickness. HTC Vive has a screen refresh rate of $90 \mathrm{~Hz}$, matched with two wireless controllers (handles), and has gesture tracking function .Started with the product, VR headset with controllers has become a standard configuration for VR hardware, and the Oculus Rift, Gear VR and Google's Daydream View successively issued their own adapter controllers following the trend[2]. Why does VR device need controllers? The reason is simple. The virtual world won't satisfy the needs of users only with vision and hearing obviously. However, if touch added, the feeling will be more real, so VR controllers become the best solution now. In addition to bringing the tactile feedback most directly, the controllers also make users operate more precisely.

\section{Project Research and Practice}

The project in this article consists of three sub-projects, virtual welding, military project display and workshop device display and purpose of each of them is different. Virtual welding is for industrial simulation education;Military and workshop project display are all for introduction.In military project display, a tank is split apart into many components to show its structure.And in workshop project display, the role can wander around to trigger the introduction canvas of the device in it which helps the wanderer know its functions quickly. The research of controllers' functions is covered in the three subprojects such as instant transfer, vibration, pickup, trigger and emission (jet), and the realization of all the functions basically cannot leave the application of VRTK plugin in Unity3D[3], which provides a lot of commonly used function of VR. When they are used, subtle difference of application methods appears among them.

Pick Up. Pickup can be realized in many ways (refer with: TABLE I, Fig. 1). One way is started with setting GameObject called pickup. And then use boolean value of kinematic attribute within its rigidbody component to keep the motion state consistency of GameObject and controllers to control grabbing. But in this method, you can only pick up a designated object one time, so experience is constrained. Another way with VRTK is based on the definitely existing scheme for the interactive GameObjects, in which one can achieve the effect of grasping any object in the scene. In virtual welding subproject, one controller is used to pick up rose petals, and the other one is regarded as a welding gun. The most important job is to add VRTK interaction scripts, rigidbody and collider components to each petal as well as controllers so as to meet the conditions of the collision. But when a petals has rigidbody component , they will have gravity property, so the petal will fall off the controllers after the trigger is released, which won't show the effect of welding. However, Unity's own function resources are rich and powerful, and function rigidbody. freezePosition() can get rid of the gravity of released GameObject, thus help fix the petals at the top of flower receptacle. Adjust the relative position of the welding gun and controller model, then stop the rendering of the controllers to make them hidden, and finally replace controllers with welding guns. 
TABLE I.

PICKUP METHODS COMPARISON

\begin{tabular}{|c|l|l|}
\hline & \multicolumn{1}{|c|}{ Rigidbody Kinematic Attribute } & \multicolumn{1}{c|}{ VRTK Pickup } \\
\hline Advantages & $\begin{array}{l}\text { Realization process is easy. } \\
\text { Object that is picked up doesn't need any other } \\
\text { components to support }\end{array}$ & Can grasp any objects in the scene \\
\hline Disadvantages & only pick up a designated object one time & $\begin{array}{l}\text { Realization process is not easy . } \\
\text { Object that is picked up needs related components }\end{array}$ \\
\hline
\end{tabular}

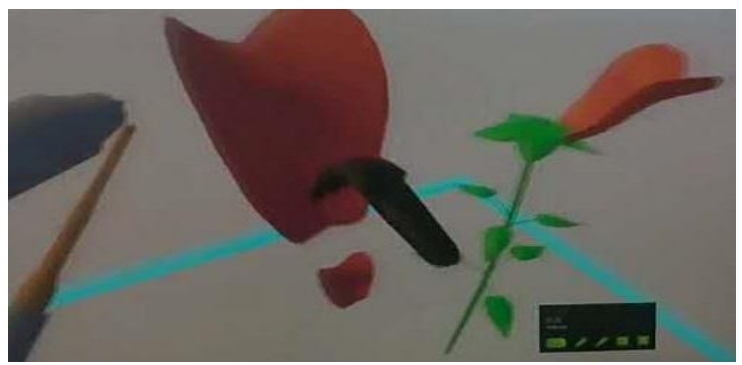

Fig. 1. Pick up.

Vibration and Emission. The fire particles whose chosen color is flame red and the maximum number of particles is 20 , are placed at the muzzle of the welding gun. By triggering the controller, emission effect is created (refer with: Fig. 2). In order to make the welding effect more lifelike, vibration effect is added into the controller, which is created when press down the trigger. As far as vibration, it should be the most direct feedback that controller can pass to the users[4]. In a lot of VR games, especially some action games, vibration makes the work more attractive. Just because of its special effects, it is applied in two subprojects: virtual welding and workshop device display. TriggerHapticPulse (int duration, ushort strength) is the core function of vibration effects, with the first parameter providing persistent timing and the second parameter for intensity of vibration. The controller vibration in this project is set to 1200 times per second. In Subproject workshop device display, one controller emits a ray (refer with: Fig. 3). Although it also belongs to the action of jet, there is fundamental difference between welding gun fire jet and this in operation principle. In virtual welding subproject, flame's spraying is to set particle system as a GameObject. Pressing down the trigger activates Play () function, and releasing the trigger running the Stop () function[4]. In workshop device display subproject, RaycastHit is set to simulate ray focus information, and the LineRenderer component is used to obtain the controller's ray effect. Both the methods are parallel to the vibration of the controllers.

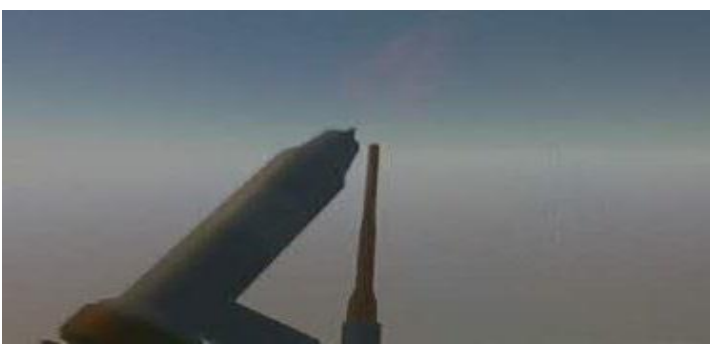

Fig. 2. Welding gun flame's spraying.

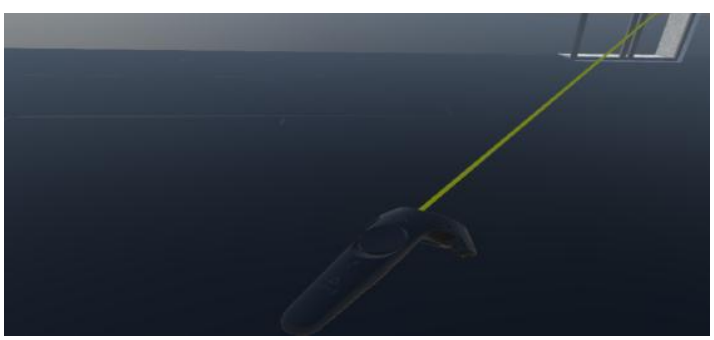

Fig. 3. Ray emission. 
Instant Teleport. The instant transfer of the controller is shown in military project display and workshop device display subprojects(refer with: Fig. 4, Fig. 5). The CameraRig prefab, which works with Unity3D to achieve VR effect[4], provides users with a PlayArea, but the activity room is limited. VRTK_Pointer script runs a core function that it can teleport the player to any place which the laser points to, just like going through the portal in the virtual world, and at the same time PlayArea in CameraRig will move along with the user. VRTK_Bezier Pointer Render script renders the parabolic laser beam, while VRTK_Straight Pointer Render script renders the linear laser beam. To complete the teleport, the place that cursor touch must have a collider component. This detail must be noticed in practice, or it won't perform the instant transfer effect.

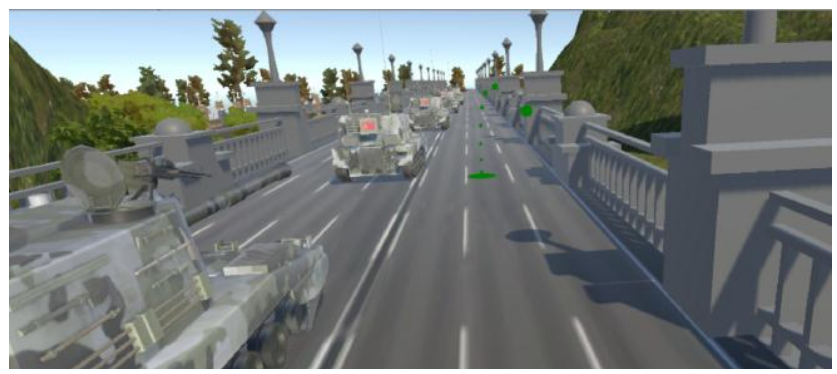

Fig. 4. Instant teleport.

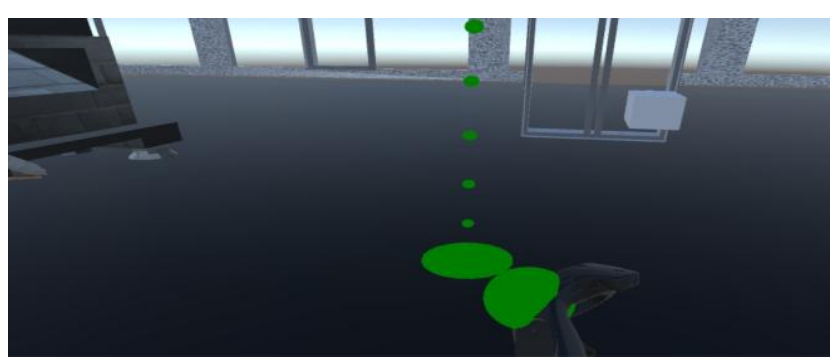

Fig. 5. Instant teleport.

Trigger and Collide. The most important functions that the military project display delivers are to split apart, assemble the tank model and rotate the every single component when the whole tank is disassembled. And all of these are realized through triggers(refer with: Fig. 6, Fig. 7). In Unity3D, there are two ways to detect collision[4], one is through colliders and the other by triggers. Trigger is an attribute in a collider. When in a collider component Is Trigger equals True[4], the collider is ignored by the physics engine body, losing its collision effect, but it has become a trigger. In the course of practice of the subprojects, sphere colliders are added to the controllers and turned into triggers. At the same time turn the GameObjects that will be touched into triggers. Put together GameObjects, with designated tags and special effects that will be triggered by function OnTriggerEnter/Stay/Exit () , and then can a series of dazzling special effects appear, such as discoloration, canvas display (refer with: Fig. 8), components dismantling, merger, rotation etc. If the triggered GameObjects extend to the particle or sound systems, the experience of virtual reality will be better. All the scripts compilation in Unity3D engine runs around the object-oriented thought, and any abstract component becomes much simpler when viewed as a GameObject.
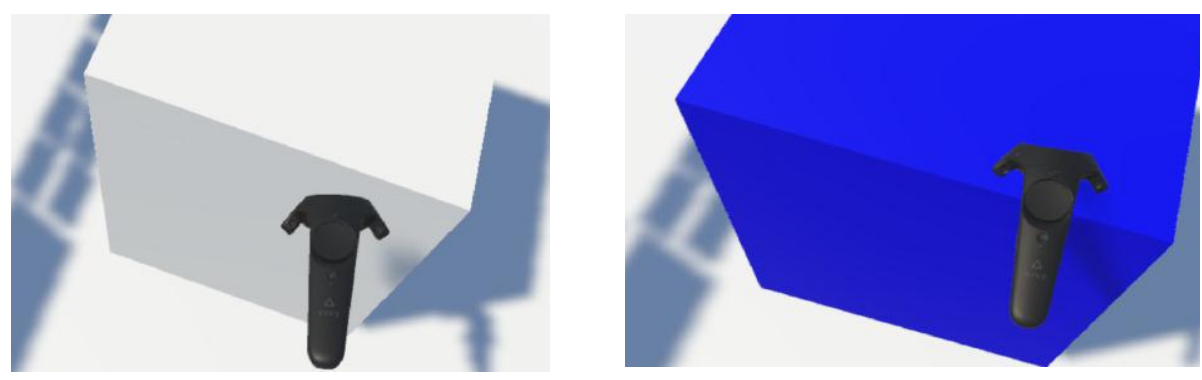

Fig. 6. Before trigger. (Left)

Fig. 7. Color change after trigger. (Right) 


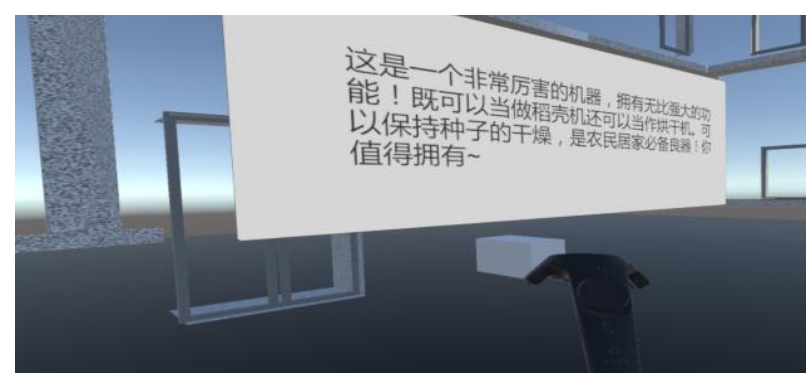

Fig. 8. Canvas display after triggered

\section{CONCLUSION}

The controller is still evolving. With the continuous development of virtual reality in the direction of mobile client, the controller will also evolve to a new look that is smaller, more convenient, more attractive and more functional. Although it will take some time to breed more advanced technology, in the context of the Information Age, virtual reality will have a more brilliant tomorrow.

ACKNOWLEDGMENT

This work was partially supported by SUSTech fund ( 05/Y01051814, 05/Y01051827,05/Y01051830, 05/Y01051839).

\section{REFERENCES}

[1] Information on http://www.iimedia.cn/39871.html

[2] Information on http://mt.sohu.com/20160818/n464884398.shtml

[3] Information on http://blog.csdn. net/kitok/article/details/52836088

[4] Information on http://answers.china.unity3d.com 